\title{
La consommation collaborative, le versant encore équivoque de l'économie de la fonctionnalité
}

Collaborative Consumption, the ambiguous aspect of the functional Economy

Isabelle Robert, Anne-Sophie Binninger et Nacima Ourahmoune

\section{(2) OpenEdition}

\section{Édition électronique}

URL : http://journals.openedition.org/developpementdurable/10222

DOI : 10.4000/developpementdurable.10222

ISSN : 1772-9971

Éditeur

Association DD\&T

\section{Référence électronique}

Isabelle Robert, Anne-Sophie Binninger et Nacima Ourahmoune, « La consommation collaborative, le versant encore équivoque de l'économie de la fonctionnalité », Développement durable et territoires [En ligne], Vol. 5, nº1 | Février 2014, mis en ligne le 04 février 2014, consulté le 10 décembre 2020. URL : http://journals.openedition.org/developpementdurable/10222 ; DOI : https://doi.org/10.4000/ developpementdurable.10222

Ce document a été généré automatiquement le 10 décembre 2020.

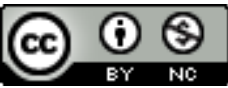

Développement Durable et Territoires est mis à disposition selon les termes de la licence Creative Commons Attribution - Pas d'Utilisation Commerciale 4.0 International. 


\title{
La consommation collaborative, le versant encore équivoque de l'économie de la fonctionnalité
}

\author{
Collaborative Consumption, the ambiguous aspect of the functional Economy
}

Isabelle Robert, Anne-Sophie Binninger et Nacima Ourahmoune

1 Encore en phase d'expérimentation il y a quelques années, l'économie de la fonctionnalité est aujourd'hui présentée comme un nouveau modèle économique d'entreprise prometteur répondant aux problématiques de développement durable. Popularisée par le Grenelle de l'environnement et médiatisée par les expériences de plusieurs entreprises menées dans des activités Business to Business (BtoB), telles que Michelin, Xerox, Elis... l'économie de la fonctionnalité s'étend depuis peu aux activités grand public. Trop souvent assimilée à la simple substitution d'un bien par un service, l'économie de la fonctionnalité nécessite d'enrichir la création de valeur en proposant aux clients des solutions globales intégrant services et usage de biens tout en consommant moins de ressources et d'énergie (Gaglio et al, 2011).

2 L'économie de la fonctionnalité a été plus particulièrement analysée sous l'angle de l'offre dans une perspective économique globale ou plus organisationnelle. Sur le plan de la demande, plusieurs travaux ont analysé les modalités de mise sur le marché de ces offres en insistant sur la nécessité de vendre au client un usage ou un résultat. Mais au-delà de ces constats généraux, les nouvelles logiques d'usage, d'accès et de consommation qui en découlent sont peu approfondies ou rejoignent la littérature sur les services (Lovelock et Gummeson 2004). Or, les marchés connaissent aujourd'hui des modifications substantielles en matière de relations à la consommation et aux biens (Belk, 2010). La consommation dite «collaborative » (Felson et Spaeth, 1978) fait partie de ces tendances qui renouvellent les logiques consommatoires via la mutualisation, l'échange, le troc ou le partage. Elle s'inscrit plus globalement dans l'économie du partage comme l'atteste le foisonnement des termes gravitant autour d'elle: covoiturage, cohousing, coworking, crowdfunding, crowdsourcing... Elle fait partie de ces nouvelles pratiques de consommation dynamisées par le développement des réseaux électroniques et des réseaux sociaux et 
aujourd'hui considérées comme révolutionnaires et dotées de nombreuses vertus: respect de l'environnement, praticité, gains économiques, simplicité, création de liens sociaux... Remettant l'usage des biens au cœur des enjeux face à la propriété individuelle (Bostman et Rogers, 2011), elle actualise les notions de partage, de prêt, de location et de services autour des produits, s'insérant alors de fait dans la réflexion autour de l'usage des produits propre à l'économie de la fonctionnalité.

Or, si l'économie de la fonctionnalité vise à réduire les externalités environnementales négatives, ces nouvelles logiques d'usage facilitées par la consommation collaborative s'intègrent-elles totalement dans ce schéma directeur? Cet article a pour objectif d'étudier l'émergence de la consommation collaborative et d'analyser sa contribution au développement durable en regard de l'économie de la fonctionnalité. En effet, la consommation collaborative est souvent présentée comme une nouvelle forme de consommation alternative, de consommation durable, s'inscrivant même dans des logiques de décroissance et «d'optimisation de la non-consommation $»^{1}$. Toutefois, son essor, via le développement des places de marché en peer to peer (pair à pair) questionne la dimension durable et l'idéologie de consommation sous-tendue. N'est-elle pas alors appréhendée comme un simple palliatif à la crise, comme un nouveau comportement de consommation émanant de l'acheteur opportuniste ("smart-shopper") voulant faire une bonne affaire, ou comme une expérience consommatoire de plus contribuant à entretenir la rivalité mimétique dans des besoins sans cesse renouvelés?

4 Cet article se propose d'analyser la consommation collaborative telle qu'elle est approchée dans la littérature. Il prend appui sur des exemples récents américains et français de consommation collaborative et sur l'analyse de 25 sites et deux blogs essentiellement français identifiés comme faisant partie de ces nouvelles approches. Il mettra en évidence dans un premier temps les fondements de la consommation collaborative puis tentera d'isoler au sein des typologies ainsi identifiées celles plus particulièrement reliées aux principes de l'économie de la fonctionnalité et leur contribution possible à la consommation durable.

\section{Les différentes formes de consommation collaborative et leur intégration dans l'économie de la fonctionnalité}

5 Dans une logique de durabilité, l'économie de la fonctionnalité qui consiste à remplacer la vente d'un bien par la vente "d'une solution adossée à une performance contractualisée et fondée sur l'usage d'un ensemble intégré de biens et services » (Gaglio et al, 2011) repose sur un découplage entre flux de matière et croissance économique et sur une intégration des externalités. Ce nouveau modèle économique doit donc contribuer à « déplacer le centre de gravité de l'économie des moyens vers les finalités, et il est de nature à permettre d'internaliser l'économie des ressources au cœur des modèles de rentabilité» (Moati, 2012: 158). Toutefois, l'économie de la fonctionnalité n'est pas une simple extension du principe d'écoefficience qui vise à accroître la productivité des ressources mais impose également de revisiter le fonctionnement linéaire des systèmes industriels classiques en introduisant le principe de circularité (Gaglio et al, 2011). Proposant une définition élargie de l'économie de la fonctionnalité, Du Tertre (2011 : 35) singularise ce modèle comme étant basé sur « la conception et la réalisation de solutions s'appuyant sur l'intégration de biens et de services de telle 
manière que cette dernière provoque un changement de niveau systémique des dimensions "fonctionnelles" et institutionnelles de l'action productive".

6 Le recentrage sur la valeur d'usage et plus globalement sur " les effets utiles que procurent, dans l'usage, l'intégration des produits ou des services" (Gaglio et al, 2011) et l'absence de transfert des droits de propriété au client constituent la clé de voute du modèle, impliquant de revisiter les modes de production, d'organisation, de rémunération des entreprises et d'initier de nouveaux comportements d'usage aussi bien au niveau des entreprises que des consommateurs. Comme le précise Lauriol (2008: 33), «l'économie de la fonctionnalité s'inscrit dans une économie de services dans laquelle le bien au sens physique du terme, est considéré comme une immobilisation (et non plus comme un "consommable") ». Elle nécessite donc de repenser la propriété et par sa nature coopérative et co-créatrice, de réexaminer les rapports de propriété sur les marchés (Rifkin, 2012). Elle doit également révolutionner l'activité productive (Moati 2012: 156), qui «s'est jusque-là concentrée sur la production d'outputs et devrait porter sur la fourniture d'outcomes ».

7 La contribution de ce modèle au développement durable repose d'une part sur l'offre à travers l'éco-conception du produit, la maximisation de sa durée de vie et l'optimisation de son taux d'utilisation (Van Niel, 2007). Son gain environnemental peut également être optimisé si ce modèle intègre dans ses fondements les principes de l'économie circulaire (Mont, 2000, Stahel, 1998, Gaglio et al, 2011...) et adopte une gestion optimisée du cycle de vie du produit (reprise des produits, remise en état, reconditionnement, réutilisation des composants pour une valorisation ultérieure et recyclage des matières...). Du côté de la demande, c'est à travers la mutualisation et l'intensité d'usage du produit que les bénéfices pour l'environnement sont visibles.

8 Au cours des dernières années, différentes stratégies d'entreprise intégrant les principes de l'économie de la fonctionnalité se sont développées en s'inspirant du concept anglosaxon dérivé de Product-Service Systems (PSS) (Goedkoop et al, 1999, Mont 2002, Mont 2004, ...) communément défini comme un ensemble intégré de produits et de services visant à satisfaire les besoins des consommateurs. Ces PSS sont répartis usuellement selon la classification suivante d'Hockerts (1999): les PSS orientés produit (offre d'un service complémentaire à la vente d'un produit), les PSS orientés usage (vente de l'usage du produit dans le cadre d'activités de location, de leasing, de mutualisation, de partage, de regroupement de clients...) et les PSS orientés résultat (satisfaction des besoins du consommateur et garantie des résultats reposant sur une combinaison de moyens et d'alternatives). Par la diversité des PSS recensés, excepté ceux orientés produits non assimilables à l'économie de la fonctionnalité, ce modèle économique offre de nombreuses opportunités stratégiques en termes d'innovations techniques et servicielles (Gaglio et al, 2011). L'économie de la fonctionnalité permet de pérenniser la relation commerciale en centrant la relation sur ce qui se joue après l'achat et non uniquement sur le moment de l'achat (Moati, 2012). Elle doit aussi contribuer à tisser des liens durables avec les clients en revisitant les relations sous le prisme de la coopération - coconception, co-production et co-évaluation - (Valeanu-Paun et Boutillier, 2012). Dans cette même logique, elle faciliterait une «fidélisation soutenable » sans que le client soit captif (Zacklad, 2010), en développant une confiance mutuelle et "un engagement volontaire explicite sur la durée» déterminée par une contractualisation orientée résultat ou modalités d'usage.

9 Les avantages de ce nouveau modèle économique s'intègrent plus directement dans des entreprises travaillant en B to B comme nous le démontre la multiplication des études de 
cas dans des secteurs diversifiés (Van Niel, 2007 ; Gaglio et al, 2011 ; Sempels et Hoffmann, 2012). Mais pour les marchés $B$ to $C$, les obstacles d'ordre psychologique et symbolique apparaissent plus contraignants à sa diffusion. En effet, comme le soulignaient Bourg et Buclet (2005: 36 ), «l'attachement des individus à la propriété, le besoin de différenciation par l'objet et le moindre respect accordé aux biens que le consommateur ne possède pas, n'auguraient pas une extension rapide de ce modèle auprès du grand public ». Pourtant la vente de l'usage d'un bien connait un certain succès dans le domaine du grand public à travers la banalisation de l'injonction «n'achetez plus, louez!». Cet essor se concrétise par le développement des sites de location sur internet et des places de marché, qui mettent en relation des propriétaires de biens et des locataires potentiels, permettant d'optimiser le taux d'usage du produit. Dans certains cas, lorsque la mutualisation de l'usage du bien est orchestrée par un médiateur - entreprise, association, agence publique -, le cycle de vie du produit peut être amélioré par une maintenance du produit permettant l'extension de sa durée de vie, par la mise en location de produits économes en consommation d'énergie, par une gestion de la fin de vie du bien ou par une modification du produit visant à accroître sa robustesse et sa durabilité (exemple du Velib'). Indéniablement fondées sur les principes de l'économie de fonctionnalité, ces nouvelles formes de vente s'arriment également au nouveau concept de consommation collaborative.

Terme inventé en 1978 par Felson et Spaeth, la consommation collaborative désignait originellement les "événements dans lesquels une ou plusieurs personnes consomment des biens ou des services économiques dans un processus qui consiste à se livrer à des activités communes " (p. 614). Concept encore émergeant au début des années 2000, le développement d'internet et des techniques collaboratives issues du Web 2.0 vont faire renaitre des comportements de consommation centrés sur l'accès, la mise en commun et le partage de biens et de services que Bostman et Rogers (2011) vont qualifier de consommation collaborative. Dans ce cadre, l'usage prévaut sur la propriété individuelle. Le partage, le prêt ou la location d'un bien contribuent à en augmenter l'intensité d'usage et les échanges directs entre particuliers sont facilités par Internet. Dans une perspective plus locale et directe, la consommation collaborative peut donner lieu à la cession des droits de propriété des objets dans des échanges basés sur le troc ou le don. Lorsqu'elle transite par le marché et repose sur la vente d'un usage, elle se rapproche de la consommation basée sur l'accès définie par Bardhi et Eckhardt (2012: 881) comme "une transaction qui peut être "intermédiée" par le marché sans qu'aucun transfert de propriété n'ait lieu». La différence structurelle avec la consommation traditionnelle se situe alors au niveau de la présentation de la transaction proposée, l'accès à l'usage de l'offre ou au résultat obtenu étant prédominant.

Indissociable de l'esprit collaboratif du web, l'essor de cette nouvelle forme de consommation s'explique selon Bostman et Rogers (2011) par les avantages issus de l'accès aux biens par opposition à la propriété, mais également par les gains économiques, spatiaux et temporels possibles. En outre, selon ces auteurs (2011: xvii), "ces systèmes offrent des avantages environnementaux signifiants en augmentant l'efficacité d'usage, en réduisant les déchets, en encourageant le développement de meilleurs produits et en épongeant le surplus créé par la surproduction et la surconsommation ». Pour faciliter l'émergence de cette nouvelle forme de collaboration, Bostman et Rogers (2011) identifient quatre principes : 1) le principe de la masse critique est une condition nécessaire pour assurer la pérennité du modèle et présuppose que le nombre de participants aux échanges soit suffisamment grand pour satisfaire les besoins des consommateurs en termes de choix, 2) la 
mutualisation des capacités excédentaires de biens, 3) la croyance en la possibilité d'une gestion collective des communs inspirée des travaux d'Ostrom (2010) et enfin, 4) la confiance entre les personnes qui est un facteur-clé de réussite dans les systèmes d'échanges et de distribution « désintermédiés ».

12 Les pratiques de consommation collaborative étant multiples de par la nature des relations entre les acteurs (partage, location, don, troc...) et des types de biens échangés, partagés et utilisés (tangibles ou intangibles), Bostman et Rogers (2011) distinguent trois grandes catégories de forme de consommation collaborative : les Product-Service Systems, les marchés de redistribution et les styles de vie collaboratif.

13 - La première catégorie se réfère aux PSS orientés usage déclinés usuellement sous la forme d'une location ou d'un leasing classique organisés par un intermédiaire (entreprise ou association) mais intègre également les locations Peer to Peer. Dans tous les cas, les droits de propriété des produits ne sont pas transférés aux consommateurs finaux. Cette forme de consommation collaborative peut être associée à la notion de « services d'usages partagés ", développée par Roy (2000: 294) qui "visent à accroître l'utilisation des biens matériels d'un système en partageant les produits dont on a besoin ». Initialement circonscrites à des fonctions de mobilité, les sites de consommation collaborative et plus spécifiquement les plates-formes d'échanges virtuelles mettant en relation des offreurs et des demandeurs se multiplient dans des domaines très variés : bricolage, électroménager, vêtements, jouets, produits de puériculture, accessoires de mode, produits de luxe ... (Cf. Tableau 1). Parmi les derniers sites crées, Partage ton frigo vise à mutualiser l'usage d'un réfrigérateur collectif et à créer des Apéros Frigo pour lutter contre le gaspillage alimentaire. Dans le cadre de la mobilité, une nouvelle place de marché dédiée à location de camping-cars entre particuliers, dénommée «Je loue mon camping-car» vient d'être récemment créée.

Outre la diversité des fonctions d'usage proposées, ces nouveaux modèles économiques attirent de nouveaux acteurs, comme en témoigne l'évolution de l'autopartage en France. Lancées par des associations et des coopératives, ces sites proposant des modes de location classique (offre de véhicules neufs dans des parkings ou stations fixes) ou des modes de location entre particuliers ont été rapidement concurrencés par les loueurs de voitures comme Avis et Hertz, les sociétés délégataires des transports publics et plus récemment par les constructeurs automobiles (lancement des plates-formes $\mathrm{Mu}$ by Peugeot et Multicity de Citroën).

14 - Le troc, le don ou l'échange de biens d'occasion entre particuliers caractérisent les marchés de redistribution. Prédominants sous leur forme d'échange marchand, le principe des plateformes web de revente entre particuliers telles que eBay, le Bon Coin, évolue vers des principes de gratuité (troc et dons). Pionnier en la matière, le site Freecycle, d'envergure internationale et déployé localement, met en relation depuis 2003 des individus qui souhaitent se débarrasser gratuitement d'objets avec des personnes qui en ont besoin. Des plateformes d'échanges basées sur le troc ont vu le jour en France comme celle dénommée " Kiditroc », dont le principe repose sur des échanges de packs de vêtements d'enfants. Certains sites pour intensifier les échanges se sont même dotés de monnaie complémentaire comme le site GuestToGuest (échange de maisons) qui a instauré un système de points pour motiver les usagers à multiplier les échanges.

15 La dernière catégorie correspond aux styles de vie dits «collaboratifs » et regroupe les formules de partage de ressources immatérielles entre particuliers comme l'espace, les compétences, le temps, l'argent. Cette catégorie comprend par exemple le partage 
d'espaces de travail (coworking), de jardins, de places de stationnement, d'hébergement comme le couchsurfing qui consiste à proposer des services d'hébergement temporaire de personne à personne. Parmi les styles de vie collaboratifs, les sites de crowfunding ou de finance participative se généralisent et permettent de lever des fonds, via les réseaux sociaux et des plateformes internet, auprès d'un groupe d'individus pour financer des projets de tout type. Dérivés de cette catégorie, on retrouve également les achats groupés directs au producteur car ils nécessitent une collaboration préalable entre un groupe de consommateurs pour conclure un accord avec un agriculteur ou petit producteur (cas des AMAP par exemple).

Tableau 1. Exemples de sites de consommation collaborative en France selon les principes de classification de Bostman et Rogers (2011)

\begin{tabular}{|c|c|c|}
\hline Product-Service Systems & Marchés de redistribution & Styles de vie collaboratifs \\
\hline $\begin{array}{l}\text { Velib' } \\
\text { Autolib' } \\
\text { Lokeo } \\
\text { Mu by Peugeot } \\
\text { Mon Joujou } \\
\text { La machine du voisin } \\
\text { Je Loue Mon Camping Car } \\
\text { Ma bonne amie (location de robes de } \\
\text { soirée haute couture) } \\
\text { Sac De Luxe } \\
\text { Mon dressing secret... }\end{array}$ & $\begin{array}{l}\text { - eBay } \\
\text { - Le Bon Coin } \\
\text { - Vestiaire de copines... } \\
\text { Troc, Dons, Échanges: } \\
\text { - Freecycle (dons) } \\
\text { - My Recycle Stuff (troc } \\
\text { circulaire) } \\
\text { - Kiditroc } \\
\text { - Homelink (échanges de } \\
\text { maisons) } \\
\text { - GuestToGuest } \\
\text { - Troc de presse... }\end{array}$ & $\begin{array}{l}\text { - Campe dans mon jardin } \\
\text { - Troc Heures de } \\
\text { Castorama } \\
\text { - Couchsurfing } \\
\text { - Ulule } \\
\text { - Babyloan } \\
\text { - La ruche } \\
\text { - Habitat coopératif } \\
\text { - Bureaux à partager } \\
\text { Achat groupé direct au } \\
\text { producteur: } \\
\text { - La ruche qui dit oui } \\
\text { - Réseau Cocagne } \\
\text { - Réseau des Amap... }\end{array}$ \\
\hline
\end{tabular}

Cet état des lieux laisse penser que la multiplication des sites de consommation collaborative dans de nombreux secteurs, et plus spécifiquement les services d'usage partagé, contribue à généraliser la promotion, l'intensité et la mutualisation de l'usage d'un bien au détriment de sa possession. Mais cette consommation s'inscrit-elle vraiment dans un changement de paradigme modifiant l'idée même de propriété ? La consommation collaborative devient-elle le reflet d'une modification majeure de la relation à l'objet et le «bras armé » d'une extension de l'économie de la fonctionnalité via la critique installée de la consommation? Permet-elle enfin de satisfaire les préoccupations environnementales des individus et leur recherche de liens sociaux? Dans les prochaines sections, nous analyserons par une étude empirique les motivations qui sous-tendent cette consommation et étudierons plus précisément les liens entre consommation collaborative et développement durable. 


\section{Méthodologie}

17 Afin de répondre à ces questions, trois méthodes d'investigation distinctes ont été retenues. La première repose sur un recensement et une analyse des données secondaires et plus particulièrement d'études et analyses académiques réalisées sur la consommation collaborative dans des contextes anglo-saxons explicitant les ressorts de cette consommation. La seconde se focalise sur une analyse de sites internet français et anglosaxons de consommation collaborative. La dernière investigue deux blogs, en adoptant une méthode de recherche qualitative. Concernant plus précisément l'analyse des sites de consommation collaborative, nous nous sommes intéressées uniquement aux sites appartenant à la première catégorie de consommation collaborative (Bostman et Rogers, 2011) correspondant aux product-service systems (PSS) orientés usage. Au sein de cette catégorie, nous avons étudié deux types de sites distincts : a) les sites dans lesquels une entreprise, une association, une agence publique... met à la disposition des clients l'accès temporaire à des biens et $\mathrm{b}$ ) les places de marché mettant en relation des particuliers directement entre eux où l'usage d'un bien proposé peut-être séquentiel ou partagé (comme le co-voiturage par exemple). Ainsi nous avons délibérément exclu les sites de redistribution et de styles de vie collaboratifs de notre champ d'analyse. Au total 25 sites (Cf. tableau 2) ont été analysés sur une période de 18 mois (février 2012-octobre 2013), la durée fut écourtée pour les sites récemment crées comme Partage ton frigo, ILokYou.... Nous avons décrypté les argumentaires marketing mis en avant par ces sites, en considérant que le site web reflète parfaitement la stratégie de communication de l'annonceur grâce aux éléments de contenu qui composent le message (argumentaire commercial, actualités diffusées, blogs, espaces personnels...). La méthodologie d'analyse de contenu a été mise en place à partir de l'exploration des différents éléments collectés sur ces sites.

Tableau 2. Recensement des sites et blogs étudiés

\begin{tabular}{ll|l} 
Sites de consommation & \\
collaborative et places de & Blogs (2) et période étudiés \\
marchés étudiées (25)
\end{tabular}




\begin{tabular}{|c|c|}
\hline $\begin{array}{l}\text { Velib' - V’Lille } \\
\text { Autolib' - Citizen Car - Zipcar } \\
\text { BlablaCar } \\
\text { Mu by Peugeot - Drivy - Je loue } \\
\text { mon camping car } \\
\text { E-loue - Lokeo - Zilok - ILokYou } \\
\text { Bricolib -Street Club } \\
\text { Mon Joujou - Ecojouet - Maman } \\
\text { loue } \\
\text { Mon dressing - Ma bonne amie - } \\
\text { Sac De Luxe } \\
\text { La machine du voisin - Baby } \\
\text { plume } \\
\text { La fabrique des lunettes - } \\
\text { Partage ton frigo }\end{array}$ & $\begin{array}{l}\text { - Blog de la consommation collaborative } \\
(11 / 07 / 2010-15 / 12 / 2013): \text { http://consocollaborative.com } \\
\text { - Blog OuiShare, la communauté de l'économie collaborative } \\
(8 / 12 / 2012-1 / 05 / 2013): \text { http://magazine.ouishare.net/fr }\end{array}$ \\
\hline
\end{tabular}

Pour les blogs, nous avons sélectionné les deux blogs les plus actifs en France. Leurs objectifs reposent sur la diffusion d'informations et l'échange entre les membres d'une communauté virtuelle adepte et/ou sensibilisée par cette nouvelle forme de consommation (Cf. tableau 2). Le forum du blog de la consommation collaborative permet aux membres de discuter de différents sujets en lien avec les actualités diffusées Dans ce cadre, nous avons analysé les articles rédigés par les membres de la communauté et les communications des membres (messages postés sur le forum de discussion, conversations sur un chat). Les articles retenus ont été sélectionnés en suivant un principe de variété et de contraste (Lincoln et Guba, 1985). 460 pages mêlant articles et messages ont été analysées et codées. Une analyse globale reprenant les données issues des sites et des blogs a été privilégiée. Une analyse de contenu manuelle a été réalisée par chacun des trois auteurs séparément, un fort consensus sur les thèmes émergeant des données a conduit à une forte fiabilité inter-codeurs.

\section{Comment la consommation collaborative s'intègre- t-elle dans la durabilité ?}

Bien que la littérature sur la consommation collaborative soit encore très embryonnaire, plusieurs auteurs (Albinsson et Perera, 2012 ; Bostman et Rogers, 2011; Gansky, 2010) s'accordent à dire qu'elle s'inscrit dans la mouvance de la consommation alternative et durable. Cette dernière rejette les modes individuels de consommation conduisant à la surconsommation et vise à les renouveler en intégrant les enjeux du développement durable. La consommation durable ${ }^{2}$ est une notion mal définie comme le souligne Reisch (1988), un « concept flou ", à géométrie variable du fait de la diversité des significations, des pratiques et des comportements qu'il recouvre (allant de l'achat de produits écologiques, équitables... à la déconsommation). Elle peut être définie comme une consommation intégrant des critères sociaux et environnementaux dans une perspective d'équité inter et intra-générationnelle (Jackson et Michaelis, 2003, Lush et al, 2011, ...). Les recherches sur la consommation durable, souvent appréhendées sous un angle 
micromarketing, se sont essentiellement focalisées sur une compréhension individuelle et rationnelle de ce nouveau consommateur, omettant d'analyser le cadre macroéconomique au sein duquel de nouveaux processus de consommation s'organisent (Kilbourne, McDonagh et Prothero, 1997, Thogersen, 2005, ...). Or, c'est dans cette perspective macrosociale que Prothero et al (2011) associent la consommation collaborative à la consommation durable considérant que les solutions collectives, potentiellement plus efficaces que les comportements individuels, peuvent être un puissant levier permettant de relever les défis du développement durable. Belk (2010) voit dans le partage une forme alternative aux modes de distribution traditionnels visant sur le plan environnemental à économiser les ressources naturelles et sur le plan social à favoriser l'esprit communautaire. Dans sa lignée, Prothero et al. (2011) estiment que la consommation collaborative, par ses valeurs intrinsèques, questionne la nature de la consommation dans une logique de soutenabilité et de durabilité. «En déplaçant les valeurs du paradigme social dominant centrées sur la propriété individuelle vers les notions de partage et de collectivité, la réduction de la consommation de biens permettrait de céder la place à une nouvelle économie qui pourrait aider à relever les problèmes comme la pollution et la consommation énergétique excessive » (Prothero et al., 2011 : 36).

\subsection{Des motivations écologiques réelles mais non prédominantes}

Tant au niveau des sites que des consommateurs, le critère écologique (réduction de l'empreinte écologique, de l'empreinte carbone, lutte contre l'obsolescence programmée des produits, lutte contre le gaspillage...) est mis en avant dans certains argumentaires de vente mais également dans les motivations exprimées par les consommateurs.

"Les objets sont ainsi plus et mieux utilisés, ce qui s'oppose à une "consommation jetable", que nous sommes nombreux à remettre en cause. Ainsi, en partageant l'usage de biens, nous sommes dans une démarche écologique évidente », extrait du site ILokYyou ${ }^{3}$.

"La Location de jouets facilite la dématérialisation et limite la prolifération des déchets. Elle devient un nouveau mode de consommation. Elle vous permet ainsi d'être plus responsable d'un point de vue environnemental tout en augmentant votre pouvoir d'achat », extrait du site Mon joujou ${ }^{4}$.

22 Toutefois, il faut noter, que ce critère est rarement cité en premier et qu'il reste très souvent couplé aux arguments économiques et/ou sociaux: "La consommation collaborative a tout pour plaire: elle permet à chacun de tirer un revenu complémentaire de ses ressources inexploitées, elle recrée du lien social entre utilisateurs de services pair-à-pair, et réduit potentiellement notre empreinte écologique. Bref, et à tous les sens du terme, l'économie du partage est une économie durable » Arthur, message posté le 7 aout 2013, blog de la consommation collaborative.

"Alors merci pour la planète, merci pour les rencontres, merci pour mon porte-monnaie!", Mathilde, message posté le 4 avril 2011sur le site BlablaCar5.

Il ressort que le motif écologique est peu prédominant dans les messages ou articles postés par les consommateurs. Il n'est exprimé que par un petit segment de consommateurs qui le couple systématiquement à d'autres critères. Ce constat corrobore les résultats de recherches récentes qui démontrent que malgré une sensibilisation grandissante des individus à l'environnement (European Commission Eurobaromètre 2008, Chang, 2011), les comportements de consommation allant dans le sens de la durabilité et motivés par des critères écologiques restent marginaux (Markulla et al., 2012, 
Gleim et al., 2013). Coté offre, le déploiement des arguments écologiques par les sites et plus particulièrement par les sites de mobilité peut être considéré à l'instar des entreprises "classiques» comme une réponse aux attentes du segment des consommateurs verts que les marketeurs tentent d'identifier et de cibler (Connolly et Prothero 2003).

Contrairement à la consommation durable, qui est couramment arrimée à la dimension environnementale (Peattie and Collins, 2009, Cohen, 2007), la consommation collaborative est dans l'esprit des usagers davantage sous-tendue par la dimension sociale du développement durable dans le sens où elle crée du lien social. Adossée à des valeurs plus altruistes, cette nouvelle consommation semble à même de développer des externalités sociales positives sur les territoires (accessibilité pour certaines catégories de personnes à des biens et services, émergence de nouvelles formes de solidarité en faveur du vivre ensemble, de la convivialité...).

\subsection{Les services collaboratifs comme réponse à une quête de lien social}

Comme l'illustrent les différentes formes de consommation collaborative présentées précédemment (excepté les plates-formes d'enchère), le partage et la mise en commun des biens sont un élément clés de la consommation collaborative, ce qui suppose que le rapport à la propriété et que l'attachement aux objets se modifient à travers deux notions clés : le partage et l'accès.

Ainsi, le partage définit par Belk (2007: 127) comme «l'acte de distribuer à d'autres ce qui nous appartient pour leur usage et/ou l'acte de recevoir ou de prendre quelque chose aux autres pour notre propre usage ", pratique confinée jusque récemment à la sphère intime (familiale ou amicale), s'étend aujourd'hui aux sphères du marché grâce aux technologies nouvelles. Belk (2010 : 725) distingue d'ailleurs deux formes de partage : sharing in et sharing out. Sharing in s'apparente "au partage limité au sein de la famille dans le sens où il présuppose, concernant le fait que la propriété soit commune, que les autres soient considérés comme une extension de soi ». En revanche Sharing out "implique de donner aux autres en dehors des limites séparant le soi et l'autre et reste plus proche du don ou de l'échange de marchandises. " La consommation collaborative telle qu'elle se développe aujourd'hui, avec une prédominance du partage des biens orchestré par une entreprise, s'inscrit davantage dans une logique de sharing out. Comme le précise Belk (2010), «Partager une voiture au sein d'un couple ou d'une famille est généralement rattaché à une logique de sharing in, en revanche partager une voiture via une organisation commerciale d'auto partage à grande échelle s'insère davantage dans une logique sharing out. ». Toutefois, les frontières entre ces deux formes de partage ne sont pas si étanches et soulèvent certaines questions posées par Belk (2010: 727), notamment: «la collaboration virtuelle peut-elle conduire à un sentiment global d'extension de soi au même titre qu'une collaboration en face à face? ".

Si le partage peut être associé à l'accès à un bien dans la mesure où il n'existe pas de transfert de propriété, Bardhi et Eckhardt (2012 :882) différencient les deux notions par "le sens perçu ou partagé de la propriété ». Le partage se caractérise par une mise en commun non-réciproque des ressources résultant d'une copropriété (Belk, 2010). En revanche, comme le soulignent Bardhi et Eckardt (2012), "dans les modèles fondés sur l'accès, il n'y a pas de transfert de propriété et de copropriété, le consommateur bénéficie tout simplement de l'accès à l'usage d'un objet ». Selon ces auteurs, la démarche altruiste et pro- 
sociale propre au partage (Belk, 2010) n'est pas constitutive des modes de consommation basés sur l'accès. Si le partage peut être considéré comme un acte communautaire dans la mesure où il relie les individus directement entre eux, crée du lien social et favorise des sentiments de solidarité (Belk, 2010) ce n'est pas le cas des modèles basés sur l'accès qui peuvent être intermédiés par des entreprises (Bardhi et Eckhard, 2012).

Tout en permettant l'accès à un bien, le désir de partage constitue bien un des ressorts de la consommation collaborative comme l'attestent les propos recensés ci-dessous sur les sites: "Nous le voyons au quotidien: la consommation collaborative n'a pas de frontière, la richesse des rencontres est sans limite et la diversité des formes de partage extraordinaire. Dans le domaine de la mobilité, nous voyons l'émergence d'une vraie révolution ", Message posté par Odile le 16 mai 2012, blog de consommation collaborative.

"Je suis persuadée que la voiture quand elle se déplace avec le covoiturage n'a pas la même couleur, elle dégage une aura bénéfique sur tout le monde, tant de partage, de bonne humeur, d'écoute, de fous rires, on s'embrasse au départ comme de vieilles connaissances qui s'apprécient et pareil à l'arrivée en se souhaitant les meilleurs choses. Imaginez toutes les voitures plus illuminées ce serait joli sur nos routes", message posté par Marie le 16 octobre 2013 sur le site BlablaCar'.

"Alors tout d'abord l'expérience "La Machine Du Voisin" peut difficilement être comparée à une expérience en laverie. Rencontrer quelqu'un de sympa et partager un café avec lui est de loin plus intéressant que le bruit des tambours ", message posté par Jean-Philippe le 21 mars 2012, issu du blog de la consommation collaborative.

"I really needed a ladder to clear out my gutters. It was great to be able to borrow one from my neighbours, rather than having to buy one - and I even got an offer of some help too!", message posté par un internaute le 21 décembre 2013 sur le site Streetclub7.

La création de lien social (faire de nouvelles rencontres) apparaît comme une des principales motivations qui encouragent certaines formes de consommation collaborative comme les pratiques de location entre particuliers ou le co-voiturage. Le partage d'un bien comme une machine à laver, un réfrigérateur ou un véhicule, fait référence à la valeur d'usage mais également à "la valeur de lien », décrite par Godbout (1992: 244) comme "ce que vaut un objet, un service, un "geste" quelconque dans l'univers des liens». Ainsi, certaines pratiques de consommation collaborative contribuent à donner aux objets une valeur distinctive fondée sur « leur capacité d'exprimer, de véhiculer, de nourrir des liens sociaux » (Godbout, 1992: 245). La dimension communautaire de cette nouvelle logique consommatoire matérialisée par le désir de partage, de complicité ou d'échange d'expériences apparait explicitement sur certains sites ou messages de consommateurs : "Fais de nouvelles rencontres en partageant, élargis ta communauté ", extrait du site Partage ton frigo ${ }^{8}$. "Le covoiturage c'est une pincée d'aventure, un gros morceau de rencontres en tout genre mais toujours sympathiques, Le covoiturage c'est un petit sursis pour la planète, Le covoiturage c'est des paysages, de la route, de la route et puis un arc-en-ciel, un éclair, un coucher de soleil, Le covoiturage(.fr) c'est une communauté au sens noble. Le covoiturage c'est la vie et c'est devenu mon nouveau compagnon de route. ", Virgile, message posté le 31 mai 2011 sur le site BlablaCar?.

30 Cette volonté de fonder une communauté peut également être impulsée par les sites euxmêmes. Ainsi, le site streetclub, plateforme sociale communautaire développée par l'enseigne de bricolage anglaise $\mathrm{B} \& \mathrm{Q}$, vise à faciliter le prêt et l'échange d'objets entre voisins mais aussi à dynamiser l'esprit de quartier en créant un réseau social local : “Ever thought how much more social, helpful and rewarding it would be if you could easily talk, share 
and plan with your street? We did too - so we created Streetclub to connect you \& your neighbours with a private 'on-line community noticeboard' that's easy and safe to use. Why not start a club, talk, share and plan with those around you, and bring back a sense of community today!" extrait du site Streetclub ${ }^{10}$.

Cette dimension communautaire renvoie aux travaux portant sur la place croissante accordée aux communautés postmodernes et au tribalisme (Maffesoli, 1988, Cova, 1997) dans nos sociétés. Ces nouvelles pratiques font ainsi écho à cette idée d'un tribaliste postmoderne qui cherche dans la consommation «(...) un moyen de former des liens avec autrui dans le cadre d'une ou de plusieurs communautés de référence, qui donnerait sens à sa vie " (Cova, 1997 : 307). Ce tribalisme est caractérisé par des entités éphémères et regroupant des individus partageant des émotions, styles de vie ou pratiques consommatoires similaires (Cova, 1997).

Toutefois, cette dimension communautaire explicite dans les propos des consommateurs diffère selon les modalités temporelles d'usage proposées par les sites et selon la nature des détenteurs de biens. Ainsi, lorsque l'usage d'un bien est partagé simultanément entre les individus (cas du covoiturage par exemple), service pouvant être assimilé à une forme de partage payant, la recherche de lien social apparait prédominante. Ce besoin prévaut également lorsque l'accès du bien se fait via une place de marché où les individus vont être mis en relation physiquement (exemple des sites tels que la machine du voisin, je loue mon camping car, Bricolib...) alors que dans les modes d'accès traditionnels fondés sur un anonymat à la fois interpersonnel et spatial, ce lien communautaire est quasiment absent. Il peut même, dans certains cas, être rejeté lorsqu'il est volontairement mis en avant par le site, comme le révèle l'étude de Bardhi et Eckart (2012) à propos de Zipcar (entreprise propriétaire de sa flotte de véhicules en BtoC).

\subsection{Des pratiques qui visent à renouveler le rapport du consommateur à l'objet}

Le développement de la consommation collaborative questionne également le rapport de l'individu à l'objet. L'achat de l'usage d'un bien et non du bien lui-même semble s'inscrire dans l'idée d'un abandon progressif des valeurs associées au matérialisme. Néanmoins, cette relation de l'individu à l'objet se révèle complexe car les objets ne satisfont pas que des fonctions utilitaires mais façonnent notre identité et reflètent notre relation avec les autres. Les biens sont révélateurs des caractéristiques de leurs propriétaires, de leurs valeurs, de leur histoire personnelle et mettent en avant leur personnalité (Richins, 1994). Les possessions matérielles contribuent à structurer pleinement l'identité de l'individu et peuvent être considérées, comme l'extension du soi dans le sens où les individus s'investissent psychologiquement dans certaines possessions (Belk, 1988). Outre cette contribution à l'identité individuelle, les objets sont également des marqueurs sociaux, révélateurs du statut social ou de l'appartenance sociale (Bourdieu, 1979) et participent de facto à l'expression de l'identité sociale de l'individu. Les biens sont également considérés comme des "réceptacles symboliques de notre mémoire» (Scholl, 2006). La possession constitue un facteur d'autonomie et de liberté (Kleine, Kleine et Allen, 1995). La propriété est fortement conditionnée par le contenu symbolique des biens acquis mais également par l'imaginaire qu'ils génèrent.

Cette littérature consacrée à l'attachement des individus aux biens a souvent conduit à associer invariablement les notions de propriété et de biens, en délaissant la question de 
la temporalité et de l'accès relatifs aux biens incarnés par la location ou le leasing (Scholl, 2006). À travers les sites étudiés, il apparait clairement que l'acquisition et l'accumulation de biens ne sont plus le mode unique de consommation et le seul moyen d'établir un lien avec l'objet (Scholl, 2006, Chen, 2009).

«Certes, il y a la crise économique, qui favorise peu l'achat et force à trouver des solutions alternatives. Mais il y a aussi une usure du désir de posséder.", Éric, message posté le 26 octobre 2010 sur le blog de la consommation collaborative.

"J'écris ces quelques lignes, encore chargée d'émotion... Je n'ai plus de véhicule depuis la semaine dernière! Je me suis mise au covoiturage il y a 2 ans et à la course à pied depuis 4 ans. A chaque fois, je n'y connaissais rien, je suis partie de zéro (surtout en course à pied !). J'aimais beaucoup le confort de mon véhicule, je me suis mise à le partager à faire plein de rencontres passionnantes! Je n'aimais pas courir, et maintenant j'en suis à 2 marathons! Merci au covoiturage pour m'avoir ouvert aux autres, et surtout pour m'avoir donné l'audace de vivre sans voiture! Comme quoi tout est possible..., " Morgane, message posté le 7 novembre 2012 sur le blog BlaBlaCar ${ }^{11}$.

Ces propos font d'abord écho à l'ébranlement de la conviction que le bonheur découle de la richesse matérielle accumulée (Belk, 1985, Jackson, 2010...). En outre, même si la valeur symbolique des biens et l'attachement des individus à ces derniers reste prédominante, l'épanouissement $\mathrm{du}$ consommateur est aujourd'hui davantage conditionné par l'expérience de consommation et le plaisir provoqué par cette expérience que par la possession du bien lui-même (Caru et Cova, 2007). Enfin, cette distanciation par rapport à l'acquisition de l'objet et par rapport à l'objet lui-même est également incarnée par l' unconsumption. Ce terme défini par Walker (1997) (cité par Albinsson et Perera, 2012) concerne "tout ce qui arrive après un acte d'acquisition ou ce qui permet de faire durer les choses, de trouver de nouvelles utilisations ou quand elles sont en fin de vie de les céder de façon responsable ». Il intègre également des pratiques renouvelées mettant en avant une seconde vie des produits tels que les achats d'occasion, la location, les achats partagés, le recyclage, le prêt, le don, la revente, le troc... Comme le soulignent Van de Walle et al. (2012), l'essor de ces nouvelles pratiques s'explique par la diversification de l'offre commerciale (brocantes, marchés aux puces, ventes, achats ou dons en ligne...) mais également par des comportements sous-tendus par des préoccupations écologiques et économiques. Dans cette même logique, les pratiques basées sur l'accès aux biens, différenciées selon les modalités temporelles d'usage et la médiation ou non d'une organisation commerciale, permettent aux consommateurs de réinventer d'autres relations avec les objets et de réduire leur appétence pour la propriété (Chen, 2009).

Ces nouvelles formes de relation à l'objet s'inscrivent également dans le phénomène de réduction accélérée de la durée de possession des objets, qui caractérise la société « moderne liquide » définie par Bauman (2013 : 7). Dans cette société, « c'est la vitesse, et non la durée qui compte». Et "dans la hiérarchie héréditaire des valeurs reconnues, le syndrome consumériste a dégradé la durée et anobli l'éphémère. Il a placé la valeur de la nouveauté au-dessus de la durabilité» (Bauman, 2013: 7 et 132). La perte de l'utilité des objets contribue à ce processus de "destruction créatrice", à la mise au rebut et à la brièveté de la durée de vie des produits. Dans une économie où la mobilité, l'éphémère, la vitesse, la flexibilité deviennent les valeurs de référence, dans une société de plus en plus liquide, posséder et accumuler, valeurs associées à la solidité, n'ont plus guère de sens.

Dans cette dynamique, les pratiques fondées sur l'accès et la mutualisation des biens pourraient illustrer une nouvelle période caractérisant notre rapport à l'objet (Durgee and O'Connor, 1995). "L'accès se révèle être un mode de consommation plus éphémère, sa 
flexibilité et son adaptabilité rendent possibles les projets d'identité liquide [sans cesse renouvelés] des consommateurs » (Bardhi et Eckhardt, 2012 : 883). En se basant sur la fonctionnalité des produits, la consommation collaborative rentre dans ce cadre de flexibilité, de légèreté et de praticité renforcées, très éloignées des objectifs environnementaux et contribue à ciseler la nouvelle recherche identitaire du consommateur.

\subsection{La consommation collaborative : une nouvelle forme de résistance du consommateur}

40 Certaines recherches menées sur les comportements des consommateurs pratiquant le troc, le partage, l'échange de biens sur internet (Nelson and al, 2007; Ozanne and Ballantine, 2010, Ballantine and Creery, 2010...) associent ces pratiques à des stratégies de résistance et de contournement du marché. Parmi ces manifestations individuelles, on constate une grande diversité des actions : plaintes, bouche à oreille négatif, défections dans des circuits parallèles, actes de dé-consommation (Schor, 1998) ou résistance créative, appelée plus communément « bricolage créatif ». Kates et Belk (2001) ont mis en évidence deux postures de résistance des consommateurs face au marché : la résistance "par la consommation» et la résistance "à la consommation». On constate que les expériences consommatoires relatées dans le cadre des achats de services d'usages partagés peuvent être considérées à la fois comme des moyens de résistance "par la consommation » contre un système marchand conventionnel en privilégiant l'échange entre pairs mais également s'inscrire dans une forme plus globale et radicale de résistance générale "à la consommation ", dans la lignée des mouvements de simplicité volontaire et de décroissance.

41 Ainsi, la consommation collaborative constitue une forme de résistance réfléchie symbolisant une volonté d'émancipation du système marchand comme le souligne Edwin sur le blog de consommation collaborative en juin 2013, "que l'on parle de "redonner du sens", le constat est le même: Internet et les logiques collaboratives qui sont basées sur son fonctionnement peuvent permettre au consommateur, au citoyen, de devenir plus acteur de sa consommation et de l'économie. ».

Le choix de partager, d'échanger voire de troquer entre particuliers souligne, «la volonté $d u$ consommateur de se réapproprier son autonomie mais également une tactique de résistance face à la normalisation des espaces marchands" (Roux, 2004). Cette économie du partage est une des voies de contournement des systèmes marchands classiques et relate une volonté de distanciation vis-à-vis de la société de consommation actuelle ${ }^{12}$. Partager un bien, privilégier son accès plutôt que son acquisition sont des actes qui peuvent être motivés par des choix idéologiques et être appréhendés comme une nouvelle forme de consumérisme politique. Ces pratiques collaboratives reflètent également une résistance "à la consommation» ou une forme d'opposition à la culture de consommation dominante (Dobscha, 1998). Ainsi, les modèles d'accès développés par des collectifs de citoyens ou des régies de quartiers comme l'usage partagé d'un jardin communautaire sont perçus par leurs créateurs et leurs participants, "comme des façons de pratiquer leur citoyenneté en dehors du marché et comme une forme de contestation de la domination du marché sur l'espace public » (Visconti et al, 2010, cités par Bardhi et Eckardt (2012). La pratique du vélo-partage est parfois motivée par ce rejet de la culture de consommation dominante symbolisée par la sacralisation de la voiture (Bardhi et al, 2012). En s'opposant plus généralement au mode de l'avoir, ces consommateurs se définissent par ce qu'ils refusent 
et vont privilégier un mode d'existence régi par l'être (Cherrier, 2009). Comme le précisent Ozanne et Ballantine (2010), le partage - sur le continuum de comportements de résistances identifiés par Fournier (1988) - ne peut être considéré comme une forme extrême de résistance active. Cependant, la mutualisation de l'usage d'un bien au même titre que l'utilisation de produits de seconde main ou l'autoproduction fait partie des stratégies de retrait de marché et de réduction de la consommation déployées par les adeptes de la simplicité volontaire. Ce sont avant tout les biens matériels qui sont récusés chez ces derniers (Ballantine and Creery, 2010). Cette résistance « à la consommation » se manifeste donc par le désir de vivre une vie moins matérialiste (Etzioni, 1998, Jackson, 2005). Les recherches menées par Ozanne et Ballantine (2010) sur les ludothèques publiques confirment que pour certaines catégories de consommateurs, le partage de biens tels que les jouets est motivé par des logiques de déconsommation. Ainsi, la mutualisation de l'usage d'un bien, dictée par cette volonté de minimiser ou de réduire la consommation, découle d'une mise à distance de l'objet voire d'une logique de désengagement affectif vis-à-vis du monde matériel pour les adeptes de la simplicité volontaire les plus radicaux.

Cette mutualisation contribue indéniablement à populariser les principes de l'économie de la fonctionnalité: elle recentre ainsi les valeurs associées aux biens sur leur fonctionnalité et dans une moindre mesure sur leur valeur ostentatoire. Si les principaux obstacles pouvant contrarier l'essor de l'économie de la fonctionnalité en BtoC reposent sur les dimensions affective, identitaire et sociale des biens, ces pratiques collaboratives, permettent d'ébranler la norme de référence du modèle de consommation construite sur l'acquisition et l'accumulation de biens et d'interroger le rapport individuel et collectif à la consommation matérielle. Ces nouveaux rapports à l'objet contribuent à désacraliser l'idée de la propriété et à revaloriser l'accès, considéré historiquement comme un mode de consommation inférieur, précaire, transitoire et limitant la liberté individuelle (Bardhi et al, 2012).

\section{Mais une logique consommatoire qui encourage le turbo-consumérisme ${ }^{13}$}

Toutefois, bien que les études et recherches portant sur les différentes formes de consommation collaborative (Albinsson et Perera, 2012; Bostman et Rogers, 2011; Gansky, 2010 ; Prothero et al, 2011, ...) mettent en avant les attitudes proenvironnementales et pro-sociales qui les caractérisent, il faut constater que les motivations individuelles utilitaristes ou d'ordre économique sont prédominantes par rapport aux valeurs altruistes, sociales et environnementales. En effet, le développement à grande échelle des plates-formes web et places de marché paraissent encourager des comportements plus individualistes qui vont à l'encontre des logiques de durabilité. Les conditions économiques incertaines, le nomadisme, les modes de vie plus éphémères, l'innovation technologique croissante, l'obsolescence technologique et psychologique des produits sont autant de motifs explicatifs du recours aux modes de consommation collaboratifs (Bardhi et al, 2012, Durgee et O'Connor, 1995). S'ajoutent à cette liste des raisons de praticité telles que la souplesse d'utilisation, la simplicité d'usage, la diminution du risque perçu engendrés par le mode locatif (Durgee et O'Connor, 1995) mais également une alternative aux contraintes de stockage et d'encombrement imposées par l'acquisition et l'accumulation de biens (véhicules, vélos par exemple). Nombreux 
sont les avis et les argumentaires des sites qui vont dans le sens de cette recherche de praticité : «Pourquoi être propriétaire de son lave-linge, de son écran plat ou de son ordinateur? Ce qui compte, c'est l'usage que l'on en fait: laver son linge, regarder la télévision, travailler ou communiquer sur son ordinateur...! Mais ce qui compte également c'est de ne pas avoir à subir les imprévus!", Extrait du site de Lokeo ${ }^{14}$. «Je vis en appartement, je vais donc pouvoir renouveler les jeux pour tous mes petits, je vais moins stocker. », message posté par Pascale le 14 janvier 2012, site Monjoujou ${ }^{15}$.

Si cette tendance se confirmait, ces comportements pourraient orienter l'offre vers des modèles serviciels basiques, déconnectés des enjeux de développement durable et déjouer les véritables innovations de rupture qu'impose l'économie de la fonctionnalité.

\subsection{Un moyen d'optimiser son pouvoir d'achat en période de crise}

Les argumentaires économiques mis en avant sur les sites de consommation collaborative ainsi que les motivations recensées sur les différents blogs apparaissent prédominants : « Le premier avantage selon moi c'est le prix. Chez un loueur professionnel les prix sont moins intéressants et si on rajoute les extras (porte vélo, draps, vaisselle, GPS, etc.) l'addition augmente rapidement (...). (...) Le propriétaire indique dans son annonce ce qu'il souhaite mettre à disposition et le prêt de ce matériel supplémentaire n'est pas facturé au locataire. Le prix du propriétaire est fixe, on sait ce que l'on paye ", Interview de la famille Guittard issu du blog JeLoueMonCampingCar ${ }^{16}$.

47 «La récession qui s'annonce va pousser les consommateurs à trouver de nouveaux moyens pour éviter de voir leur pouvoir d'achat baisser. Cela va forcément profiter a de nouveaux modèles économiques, les plus rapides à trouver la bonne solution pourrait profiter de la crise » message posté par Séménou le 14 juin 2013 sur le blog de consommation collaborative.

«E-loue est une plateforme Internet simple et performante permettant à n'importe quel particulier ou professionnel de mettre en location ses objets, et chercher des objets à louer. Leur premier objectif est: l'augmentation du pouvoir d'achat (autant des propriétaires que des locataires) qui font des économies et gagnent de l'argent. ", extrait du site Loue.com ${ }^{17}$.

Ces propos dominants dans les discours et les dernières statistiques publiées sur les pratiques de consommation collaborative (ADEME, 2013 ${ }^{18}$, OBSCOLO, 2012 ${ }^{19}$, IFOP, 2011 ${ }^{20}$ ) renforcent l'idée de la prééminence des motifs économiques (Bardhi et Eckardt, 2012; Steininger et al, 1996 ; Durgee et O'Connor, 1995 ; Katsev, 2003 ; Meikamp, 1998).

Même si ces pratiques s'insèrent également dans des logiques de consommation durable au même titre que les achats de produits d'occasion ou les achats en circuits-courts, elles sont conditionnées par l'opportunité financière qu'elles représentent et par l'immuable quête du pouvoir d'achat; ce qui reste d'ailleurs concomitant avec l'ancrage de la crise économique. Comme le précisent Cheshire et al. (2010), l'engouement pour les modes basés sur l'accès résulte de l'augmentation des coûts d'acquisition, des incertitudes économiques et sociales, de l'instabilité des relations sociales qui contribuent à éclater la cellule familiale et donc à réduire le pouvoir d'achat. Les économies financières et la reprise en main du contrôle de la consommation devancent désormais les préoccupations écologiques mises en avant lors du lancement des premières organisations d'autopartage (Katsev, 2003). Selon un sondage IFOP ${ }^{21}$ sur l'auto-partage réalisé en 2011, la location d'un véhicule via une plateforme communautaire, comme CitizenCar s'explique à hauteur de 56 \% par une volonté de réduction du budget alloué à l'automobile. 
51 Ces nouvelles pratiques de consommation collaboratives s'insèrent donc dans les comportements de consommation en lien direct avec la crise économique que sont le "smart shopping" (achat malin) (Schindler, 1989) et le "wise shopping" (achat réfléchi). Djelassi et al. (2009) distinguent ces nouveaux comportements de consommation en fonction de leur caractère offensif et défensif. Dans le cadre du "smart shopping", l'optimisation du pouvoir d'achat est systématiquement recherchée par la chasse aux bonnes affaires et le raisonnement en termes de gains est privilégié. À l'inverse de cette stratégie offensive, la démarche de "wise shopping», répondant à un besoin de sécurité vise davantage à « maitriser les dépenses en utilisant différentes tactiques destinées d'une part à restreindre les achats et d'autre part à diminuer le coût des produits achetés " (Djelassi et al, 2009 : 38).

Tableau 3. Distinction entre les comportements de Smart shopping et les comportements de Wise shopping

\begin{tabular}{|l|l|l|}
\hline & Smart shopping & Wise shopping \\
\hline Objectif & $\begin{array}{l}\text { Assouvir son envie de consommer } \\
\text { en dépensant le moins possible. } \\
\text { Recherche active de la «bonne } \\
\text { affaire» }\end{array}$ & $\begin{array}{l}\text { Maitriser ses dépenses pour respecter le } \\
\text { budget défini au préalable (quitte à } \\
\text { réduire sa consommation) }\end{array}$ \\
\hline $\begin{array}{l}\text { Rôle } \\
\text { consommateur }\end{array}$ & $\begin{array}{l}\text { Actif: temps et énergie } \\
\text { considérables consacrés à la } \\
\text { recherche de «bonnes affaires ", } \\
\text { optimisation des achats, réflexion } \\
\text { sur l'utilisation du système à son } \\
\text { profit }\end{array}$ & $\begin{array}{l}\text { Actif et critique : réflexion sur son mode } \\
\text { d'achat et de consommation, maitrise de } \\
\text { ses achats. Principaux comportements : } \\
\text { acheter moins cher mais également } \\
\text { moins, chercher à éviter les tentations }\end{array}$ \\
\hline
\end{tabular}

Source : Djelassi S., Collin-Lachaud I. et Odou P., 2009

La multiplication des sites de consommation collaborative conforte de façon évidente ces deux types de comportements. La démarche du "wise shopper", réfléchie, pragmatique et critique semble davantage en phase avec la philosophie sous-jacente des services d'usages partagés centrés sur les fonctionnalités du produit (exemples du vélo-partage, de l'autopartage). Comme le précisent Djelassi et al. (2009), dans le cadre de cette démarche plus raisonnée, le consommateur tend à contrôler et à restreindre ses dépenses en arbitrant entre différents canaux de distribution. Les nouveaux modes d'accès et de partage viennent élargir les solutions permettant d'ajuster sa consommation. Cependant, l'extension tout azimut des services de consommation collaborative à des produits technologiques ou plus sensibles au phénomène de mode (vêtements, accessoires de mode, informatique, .... $)^{22}$ laisse présager le développement de stratégies plus opportunistes, de type "smart shopper". À l'instar des sites d'échanges proposant des produits de seconde main, comme eBay (Denegri-Knott 2011), les sites de consommation collaborative ne cessent d'offrir aux consommateurs une multiplication des opportunités d'usage, suscitant le désir et favorisant des comportements utilitaristes, et détournent les outils de la société de consommation à leur profit. Si ce comportement de "smart shopper" s'impose, les économies réalisées et considérées comme des gains nets, peuvent être réinvesties dans d'autres dépenses. À l'instar de l'effet rebond lié à la frugalité et 
souligné par Schneider (2009), la baisse des dépenses facilitée par les services d'usages partagés peuvent générer un rebond de consommation vers d'autres produits. Ainsi, les effets écologiques induits par la diminution du nombre de produits fabriqués et la substitution de la vente d'un bien à la vente mutualisée d'un usage peuvent se trouver anéantis par ce rebond de dépenses qui peuvent être à leur tour matérielles et polluantes. Si cette hypothèse issue de l'analyse des sites mérite d'être approfondie dans des recherches futures, la consommation collaborative permet indéniablement d'optimiser le pouvoir d'achat.

\subsection{Une consommation qui contribue à accélérer le cycle du désir}

53 L'affirmation récente de P. Moati (2013) ${ }^{23}$ révélant que «la consommation collaborative est aussi une manière d'hyperconsommer" rappelle une problématique déjà soulevée par certains auteurs (Denegri-Knott and Molesworth, 2009 ; Denegri-Knott 2011) à propos de l'échange de produits de seconde main sur des plates-formes internet, et qui accélèrerait le désir de consommation. En effet, un site comme eBay «ne cesse de signaler des nouvelles possibilités de trouver des biens désirés, il accélère les cycles de révélations, caractérisées par des surprises et des opportunités inattendues où les produits désirés peuvent être acquis rapidement " (Denegri-Knott, 2011 : 373). Les plates-formes d'échanges de produits de seconde vie ne cessent d'accélérer le désir (Belk et al, 2003), le cercle vicieux du désir « désir - acquisition - utilisation - désillusion - désir renouvelé » en édifiant le " culte de la nouvelle ", grâce à un renouvellement continu des produits. Tout comme les plates-formes d'échanges, les Product-Service Systems et les places de marché fournissent une offre pléthorique et variée de produits et de services et permettent aux consommateurs de vivre des expériences émotionnelles originales et récréatives. La consommation collaborative répond à cet hédonisme expérientiel (Lipovetsky, 2006) dans la mesure où l'accès temporaire des biens est une occasion de vivre de nouvelles expériences (Durgee et O'Connor, 1995). Elle contribue à utiliser l'objet pour sa valeur expérientielle et distractive. Cette consommation, qui s'extrait des formes de distribution ordinaires amène à vivre des expériences ludiques, similaires à celles générées par eBay, qui sont procurées «par les activités de surveillance et de recherche et exaltées par l'espoir de trouver l'élément désiré" (Denegri-Knott, 2011).

Par cette accélération du désir générée par la simplicité d'usage, par le renouvellement continu des produits (proposés par les sites de vêtements, d'accessoires de modes, d'électroménager...) et donc par le plaisir de la nouveauté, ces services collaboratifs perpétuent la consommation répétitive de produits qui sont régulièrement rejetés du fait de leur obsolescence technologique ou psychologique comme le démontrent les discours suivants :

"J'adore le concept. Plus de jouet partout dans la maison. Quel bonheur. Les enfants se régalent avec des nouveaux jouets tous les mois ", message posté par Vanessa le 6 juillet 2010 sur le site Mon joujou ${ }^{24}$.

«Nouvelles technologies: la fin de la frustration. À peine l'emballage défait, le produit est presque déjà dépassé et rendu obsolète par l'offre renouvelée en permanence par les marques. Quand un investissement aussi conséquent rime avec insatisfaction, Mieux vaut louer! Lokéo, c'est aussi se faire plaisir! La location est un moyen pratique d'accéder à des produits à la pointe de la technologie (home cinéma, ordinateur, ...) sans avoir à investir directement une grosse somme d'argent. En plus, à la fin de votre location, vous pouvez changer de produit pour une version plus 
récente Lokéo agit en facilitateur d'accès aux produits dernier cri en électroménager, image et multimédia : alors, faites-vous plaisir! », extrait du site de Lokeo. ${ }^{25}$

«Louer des jouets chez EcoJouets, c'est avoir la certitude de recevoir des jouets de qualité, que vous pourrez choisir parmi une gamme de "best-seller ». Fini les tracas liés au stockage ou à l'obsolescence, lorsque votre forfait expire, vous nous renvoyez les jouets", extrait du site Ecojouet. ${ }^{26}$

Facilités par la reprise des produits et/ou par la circulation rapide des biens, les ProductService Systems et les places de marché encouragent le cycle usage - frustration renouvellement. L'obsolescence psychologique, provoquée par la succession de modifications mineures en termes de fonctionnalité ou d'esthétisme est la cause fréquente d'une consommation répétitive des biens tels que les vêtements, voitures, appareils électroménagers etc.. (Sheth, 2010). Elle devient un argument commercial exploité par les sites de consommation collaborative (extraits ci-dessus).

57 Cette consommation addictive, créée par ces expériences ludiques et par ce besoin de nouveauté a dégradé la durée en accélérant le cycle du désir. Selon Bauman (2013: 132), "le syndrome consumériste a placé la valeur de la nouveauté au-dessus de celle de la durabilité. (... ) il a en outre réduit le fossé séparant l'utilité et la désirabilité des biens de leur inutilité et de leur rejet ». À travers les sites étudiés, il apparait clairement que certains services collaboratifs vont à l'encontre des principes de durabilité.

\subsection{Des pratiques qui répondent aux projets identitaires des consommateurs}

58 Concourant à renforcer l'idéologie de l'hyperconsommation par ce besoin récurrent de renouvellement (Lipovetsky, 2006), la consommation collaborative peut également être associée à l'idée de consommation d'aspiration, initialement analysée par Veblen (1899) à travers le concept de consommation ostentatoire. L'aspiration à consommer des biens financièrement inaccessibles à l'accession est un argument récurrent dans le verbatim recueilli ainsi que dans les études de ces nouveaux modes d'accès (Bardhi et al, 2012; Durgee et O' Connor, 1995) : «La mission de l'entreprise : permettre à un internaute de louer des objets qu'il n'aurait pas pu financièrement s'acheter (technologie, luxe...) », extrait du site E-loue 27.

59 «Aujourd'hui Mabonneamie vous propose plus de 200 robes haute-couture à louer, mais aussi manteaux habillés, étoles, sacs, bijoux... tout pour trouver LA tenue qu'il vous faut. Et bien entendu Mabonneamie vous conseille, vous guide, vous rassure et même vous donne envie d'oser! Festival de Cannes: Vous vous rendez au festival de Cannes et vous ne savez quoi porter pour la fameuse montée des marches? Pas de panique! Robes longues, bijoux, pochettes, fourrures et autres accessoires prenez vite rendez-vous chez Ma bonne amie pour être parfaite sur tapis rouge.", extrait du site Ma bonne Amie ${ }^{28}$

60 "Lunettes à louer cherchent propriétaire d'un soir pour partager look d'enfer. Parmi une sélection de 60 modèles de lunettes solaires et de vue dans l'air du temps et signées par les plus grands designers de mode comme de jeunes créateurs, les caméléons de la mode en quête de l'accessoire parfait n'ont plus qu'à louer selon leurs envies et à tout petits prix l'objet du désir!», extrait du site La Fabrique de lunettes ${ }^{29}$.

61 Cette possibilité d'accéder à des biens jusqu'ici incompatibles avec les contraintes budgétaires renforce la tendance analysée par Schor (1998) de déplacement vers le haut 
des aspirations et d'étirement vertical des désirs des consommateurs vers des groupes de référence ayant des pouvoirs d'achat très élevés, conduisant à créer une culture nationale du luxe (Bardhi et al, 2012).

Au-delà du registre de la consommation ostentatoire faisant référence à la consommation "pour l'autre ", ces nouvelles pratiques contribuent à façonner les projets identitaires multiples des consommateurs. Dans le cas de l'auto-partage (Bardhi et al, 2012), les utilisateurs expriment leurs désirs d'expérimenter différents modèles de voitures afin de se libérer des contraintes liées à la propriété mais surtout afin d'accéder à une plus grande liberté et flexibilité de style de vie. Ainsi, selon les deux auteurs, "l'accès permet aux consommateurs de poursuivre leurs projets d'identité postmoderne en changeant de modèles de véhicules selon le style de vie recherché». Ces conclusions rejoignent celles de Durgee et $\mathrm{O}^{\prime}$ Connor (1995) qui appréhendaient la location comme un outil d'auto-exploration de soi et d'auto-projection. Par leur choix et leur simplicité d'accès, ces nouveaux services collaboratifs permettent de vivre des expériences sans cesse renouvelées où l'objet devient temporairement l'accessoire d'un style de vie flexible. Certains sites exploitent cette quête identitaire en jouant non seulement sur cette consommation d'aspiration mais également sur la diversité et la liberté de styles de vie qu'offre ce mode d'accès.

Considéré par Rifkin (2000) comme l'avènement d'un nouvel âge, l'accès centré sur la valeur d'usage des biens permet finalement de pallier les défaillances du modèle traditionnel dominé par la propriété en intégrant les nouvelles dimensions temporelles imposées par la société moderne liquide (Bauman, 2013), tout en conservant les valeurs de signes (Baudrillard, 1968) conférées par la possession temporaire des biens et tout en permettant aux consommateur de vivre pleinement cette consommation expérientielle. Sous ce prisme identitaire et récréatif, la consommation collaborative contribue donc à l'hyperconsommation.

\section{Représentation des interactions entre les logiques de consommation collaborative et durable}

64 L'analyse de la littérature, des blogs de consommation collaborative et des argumentaires commerciaux extraits des sites fait ressortir deux axes qui permettent de situer les différentes ressorts comportementaux relatifs à la consommation collaborative (voir figure 1) : un axe relatif aux valeurs avec d'un côté les valeurs collectives, altruistes et de l'autre les valeurs individuelles et centrées sur soi, un axe relatif à l'attitude vis-à-vis de la consommation avec d'une part une attitude volontaire et proactive et de l'autre, une attitude plutôt subie et défensive. Nous constatons d'abord que la consommation collaborative s'envisage prioritairement comme une attitude proactive avec des consommateurs heureux de modifier leurs habitudes et qu'elle s'envisage peu de façon défensive. Il faut noter également que ces ressorts ne sont pas exclusifs les uns des autres, qu'ils ont au contraire tendance à se combiner et s'associer dans les propos des consommateurs. Ainsi, la juxtaposition des motivations écologiques, économiques et sociales est récurrente, notamment dans le domaine de la mobilité. Si indéniablement nos résultats font ressortir la prééminence des motivations individualistes et utilitaristes, des motivations plus durables viennent donc s'ajouter à travers des attitudes critiques, de résistance, de recherche de lien social et de volonté d'être plus écologique D'autres attitudes plus traditionnelles comme le "wise shopping" peuvent également s'adosser à des critères de consommation durable, tout comme la nouvelle relation à l'objet qui peut 
s'interpréter dans un sens de surconsommation expérientielle mais aussi de volonté de moins consommer et moins posséder. Le schéma ci-dessous permet de situer l'ensemble de ces ressorts.

Figure 1. Ressorts et motivations associés à la consommation collaborative

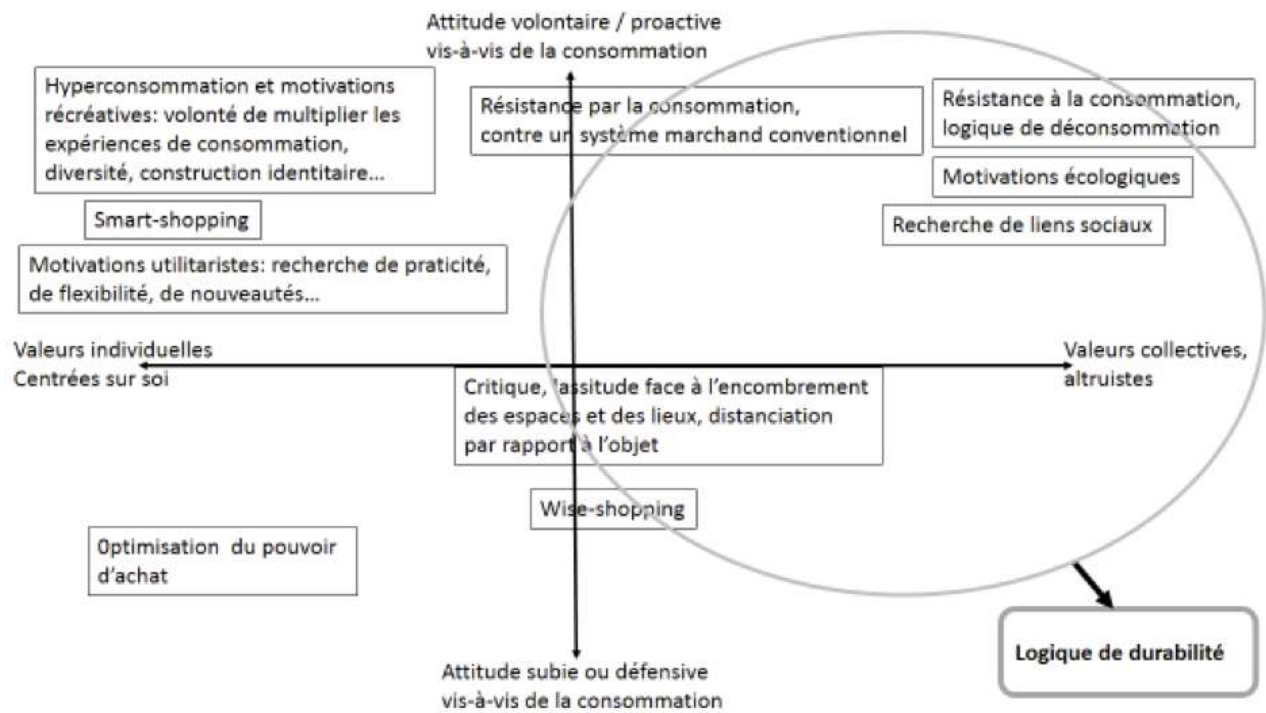

En partant de ces résultats, plusieurs pistes semblent nécessaires pour faciliter un rapprochement plus direct entre la consommation collaborative et la consommation durable. En premier lieu, il parait indispensable que les opérateurs (fabricants, sites, intermédiaires) soient proactifs et participent à la construction d'offres "durables". Ainsi, en couplant, les avantages de l'économie de la fonctionnalité (mutualisation et intensité des usages) à l'économie circulaire (extension de la durée de vie du produit, recyclage en fin de vie du produit, reconditionnement ...) ou en mettant en évidence les externalités sociales positives sur le territoire de ce nouveau modèle économique (création de liens sociaux, développement de services locaux multi-acteurs, nouvelles formes de solidarités, ...), ils contribueraient à asseoir ce modèle économique sur des bases écologiques, sociales et sociétales qui en assureraient la pérennité. D’autres orientations peuvent également permettre d'ancrer plus directement ce modèle sur ces bases. Elles sont en lien avec la nature et l'organisation de «l'offreur " de services et la nature des produits et services concernés. Ainsi, la nature du producteur à travers sa crédibilité en termes de développement durable, ses pratiques d'éco-conception, ses liens avec l'économie sociale et solidaire, ses dispositifs pédagogiques etc., ainsi que la nature de l'offre proposée centrée sur l'usage des produits, sur une durabilité effective, renforcée par le type d'informations environnementales délivrées etc. doivent générer une relation plus «naturelle» entre consommation collaborative et durable (cf. tableau 4). De même, au niveau de l'organisation orchestrant la vente de l'usage, la présence ou non d'intermédiaire, la nature de l'intermédiaire (entreprise, association, coopérative...) mais également les modalités d'accès ainsi que les services associés à la vente d'usage (création d'une communauté, ventes d'usage couplées à des trocs de biens et de services, ...) apparaissent décisifs pour contribuer à rendre la consommation collaborative plus environnementale et sociale. 
Tableau 4. Identification des variables de l'offre pouvant générer une consommation collaborative durable

\begin{tabular}{|c|c|}
\hline $\begin{array}{l}\text { Variables relatives au fabricant du bien dans le } \\
\text { cadre d'une logique d'accès intermédié : }\end{array}$ & $\begin{array}{l}\text { Variables relatives aux produits et aux } \\
\text { services proposés par le fabricant: }\end{array}$ \\
\hline $\begin{array}{l}\text { - Nature et identité du fabricant : local, national, } \\
\text { PME,...; } \\
\text { - Mise en place de démarches d'éco-conception } \\
\text { (fabrication, process...), d'économie circulaire, } \\
\text { d'éco-socio-conception; } \\
\text {-Entreprise insérée dans l'économie sociale et } \\
\text { solidaire; } \\
\text { - Développement d'une image RSE de } \\
\text { l'entreprise; } \\
\text { - Développement d'une image de marque relative } \\
\text { à la durabilité (robustesse, qualité, engagement } \\
\text { dans les filières de production, respect des } \\
\text { producteurs de matières premières, obtention de } \\
\text { labels...) }\end{array}$ & $\begin{array}{l}\text {-Type d'usage proposé: mobilité, habitat, } \\
\text { bricolage...; } \\
\text {-Dispositifs écologiques mis en avant } \\
\text { (informations environnementales : } \\
\text { composants, empreinte écologique du } \\
\text { produit, analyse du cycle de vie, labélisation, } \\
\text {..); } \\
\text { - Organisation structurée et visible du SAV, } \\
\text { de la réparation, du réemploi et du recyclage } \\
\text { des produits en fin de vie; } \\
\text { - Mise en avant des informations sociétales : } \\
\text { traçabilité sociale des produits, origine des } \\
\text { produits,... }\end{array}$ \\
\hline $\begin{array}{l}\text { Variables relatives aux communautés ou } \\
\text { entreprises orchestrant la vente de l'usage: }\end{array}$ & $\begin{array}{l}\text { Variables relatives aux services proposés par } \\
\text { le vendeur: }\end{array}$ \\
\hline $\begin{array}{l}\text { - Présence ou non d'intermédiaires; } \\
\text { - Organisation : peer to peer, association, régie } \\
\text { de quartier, coopérative, entreprise...; } \\
\text { - origine géographique du site : local, national...; } \\
\text { - Transparence du mode de rémunération du } \\
\text { site ; } \\
\text { - Modalités du partage : simultané, séquentiel, } \\
\text { courte durée...; } \\
\text { - Inscription dans une démarche partie prenante } \\
\text { (lien avec les collectivités territoriales, } \\
\text { associations de résidents, de consommateurs...) }\end{array}$ & $\begin{array}{l}\text { - vente de l'usage associée à des systèmes de } \\
\text { troc, de dons de biens; } \\
\text { - Mise en valeur du lien social, de la } \\
\text { communauté, de la dimension festive; } \\
\text { - Services s'inscrivant dans une démarche } \\
\text { territoriale (mobilité durable, habitat } \\
\text { durable...) en lien avec les acteurs locaux; } \\
\text { - Proposition de services d'entraide, } \\
\text { d'expertise...; } \\
\text { - Mise en avant sur le site d'autres modalités } \\
\text { de partage (exemple des jardins partagés), } \\
\text { d'échange de services (exemple des } \\
\text { Accorderies en France)... }\end{array}$ \\
\hline
\end{tabular}

L'ensemble de ces variables identifiées constitue une première trame qui mérite d'être analysée de façon plus approfondie dans des travaux ultérieurs. Ceux-ci devront permettre d'expliciter comment elles peuvent générer une conscience plus directe des liens entre consommation collaborative et durable et des changements comportementaux de la part des consommateurs. 


\section{Conclusion}

67 Par cet article, nous questionnons les relations entre les principes de durabilité de l'économie de la fonctionnalité et de la consommation collaborative dans le sens d'un découplage création de richesses/matière et d'un développement des externalités sociales positives. Indéniablement, la consommation collaborative contribue à démocratiser les modèles économiques orientés usage et permet d'atténuer les obstacles psychologiques inhérents au rapport à la possession des biens. En ce sens, elle permet d'expérimenter cette transition de la propriété à l'accès et au partage et permet au consommateur de se distancier de l'objet lui-même en construisant de nouveaux rapports individuels et sociaux à l'objet. Elle déclasse les valeurs conférées usuellement à la propriété (indépendance personnelle, sécurité, liberté ....) et dote de valeurs positives les modèles basés sur l'accès (simplicité, tranquillité, flexibilité, réduction du risque perçu...). Ainsi, ces nouveaux modèles ébranlent la suprématie incontestée de la logique de propriété.

La consommation collaborative qui réinvente de nouvelles formes de relations entre consommateurs et producteurs et entre consommateurs permet aussi de sortir des logiques économiques, organisationnelles et productives descendantes souvent propres à l'économie de la fonctionnalité qui en oubliait le consommateur. Elle confirme également l'autonomisation croissante de ce consommateur, son désir de « redevenir acteur » dans le processus de consommation (Lallement, 2010) et sa méfiance grandissante envers les modèles préétablis imposés par les marques et les distributeurs. Ce nouveau mode de partage de biens tangibles et intangibles répond également à une nouvelle logique de participer, de co-produire ensemble et à une volonté de réduire la médiation entre production et consommation.

Mais elle reste révélatrice également de la dualité de ce consommateur, enclin à être à la fois en dehors et dans le système. Comme le précise Lallement (2010:36), «chez tous ces consommateurs d'un nouveau type se joue certes une méfiance vis à vis du monde marchand mais dans le même temps le désir de participer à l'échange marchand. Plus encore peut-être, leur rejet des systèmes d'échanges existants traduit une volonté d'être acteur dans l'échange. En quelque sorte c'est comme s'il s'agissait surtout d'entrer, en tant qu'acteur, dans la vaste ronde des échanges. C'est toujours quelque part consommer ; c'est surtout être acteur du monde marchand ". Comme cet article l'a montré, la consommation collaborative va dans le sens du renforcement de l'hédonisme expérientiel du consommateur et peut combler son avidité à vivre des mini-expériences (Lipovetsky, 2006) à la fois émotionnelles et ludiques. Elle peut alors contribuer à assouvir le pouvoir de consommation des citoyens dans un processus d'optimisation de leur pouvoir d'achat.

70 La consommation collaborative alimente le débat qui se situe autour de la question clé de la consommation durable : le développement durable exige-t-il de consommer autrement ou de consommer moins (Jackson et Michaelis, 2003) ? Elle s'inscrit dans des schémas de consommation durable mettant l'accent sur "consuming more efficiently" as opposed to "consuming less", qui s'apparente à "the ecological modernization of consumption" (Spaargaren, 2000). Modèle alternatif possible de la consommation, elle ne pourra contribuer à installer l'économie de la fonctionnalité que si le paradigme social dominant caractérisé par une croyance en une croissance matérielle indéfinie et le mythe de la consommation illimitée sont interrogés (Prothero et al, 2011). Les valeurs portées par cette hyperconsommation ont radicalement modifié le rapport à la consommation en la 
déconnectant de l'environnement. Comme le rappelle Dolan (2002), le sentiment de supériorité de l'homme sur la nature n'est pas "simplement le produit de l'imagination de l'individu mais le résultat des interdépendances tant entre les hommes et la nature qu'entre les différents groupes sociaux au sein de la société».

Envisager l'intériorisation du concept de développement durable dans la consommation nécessite une modification profonde des valeurs sociales et identitaires de celle-ci, qui ne pourra faire l'économie d'une réflexion sur la frugalité, la déconsommation ou la nonconsommation. Pour s'ancrer dans une logique de soutenabilité forte, la consommation collaborative doit non seulement interroger la dimension politique de la consommation mais être soutenue par une économie de la fonctionnalité qui s'inscrit pleinement dans une dynamique macroéconomique durable (Du Tertre, 2011), s'émancipant des modes de régulation actuels.

\section{BIBLIOGRAPHIE}

Albinsson P. A. and Perera B. Y., 2012, "Alternative marketplaces in the $21^{\text {st }}$ century : Building community through sharing events", Journal of consumer Behaviour, 11, p. 303-315.

Ballantine P.X. and Creery S., 2010, "The consumption and disposition behaviour of voluntary simplifiers, Journal of Consumer Behaviour, 9, p. 45-56.

Baudrillard J., 1970, La société de consommation, Paris, Éditions Denoël.

Bauman Z., 2013, La vie liquide, Paris, Éditions Fayard.

Bardhi F. and Eckhardt G.M., 2012, “Access-Based Consumption : The Case of car sharing”, Journal of Consumer Research, 39, 4, p. 881-898.

Bardhi F., Eckhardt G.M and Arnould E.J, 2012, "Liquid Relationship to Possessions", Journal of Consumer Research, 39, p.510-529.

Belk R. W., 1985, "Materialism : Trait aspects of living in the material world”, Journal of Consumer Research, 12, p. 265-280.

Belk R.W., 1988, "Possessions and the Extended Self," Journal of Consumer Research, 15, p. 139-68.

Belk R.W., 2007, “Why Not Share Rather than Own ?" Annals of the American Academy of Political and Social Science, 611 (1), p. 126-40.

Belk R. W., 2010, “Sharing”, Journal of Consumer Research, 35, p. 712-734.

Belk R.W., Ger G ; and Askegaard S., 2003, "The Fire of Desire : A Multisited Inquiry into consumer Passion," Journal of Consumer Research, 30, p. 326-351.

Bostman R. and Rogers R., 2011, What's Mine is Yours. How Collaborative Consumption is Changing The Way We Live, London, Ed Collins.

Bourdieu P., 1979, La distinction, critique sociale du jugement. Paris, Les éditions de Minuit.

Bourg D. et Buclet N., 2005, « L'économie de fonctionnalité. Changer la consommation dans le sens du développement durable », Futuribles 313, p. 27-37. 
Caru A. and Cova B., 2007, Consuming Experiences, London, Routledge.

Chang C., 2011, "Feeling Ambivalent About Going Green : Implications for Green Advertising Processing”, Journal of Advertising, 40 (4), p. 19-31.

Cheshire L., Walters P. and Rosenblatt T., 2010, "The Politics of Housing Consumption: Renters as Flawed Consumers on a Master Planned Estate," Urban Studies, 47 (12), p. 2597-2614.

Chen. Y., 2009, "Possession and Access: Consumer Desires and Value Perceptions Regarding Contemporary Art Collection and Exhibit Visits", Journal of Consumer Research, 35, p. 925-40.

Cherrier H., 2009, “Anti-consumption discourses and consumer-resistant identities”, Journal of Business Research, 62, p. 181-190.

Cohen, M. J., 2007, “Consumer credit, household financial management and sustainable consumption", International Journal of Consumer Studies, 31, p. 57-65.

Connolly J. and Prothero A., 2003, "Sustainable consumption: consumption, consumers and the commodity discourse", Consumption Markets \& Culture, 6 :4, p. 275-291.

Cova B., 1997, "Community and consumption. Towards a definition of the "linking value" of product or services", European Journal of Marketing, 31, 3/4, p. 297-316.

Denegri-Knott J. and Molesworth M. , 2009, "I'll Sell this and I'll Buy Them That: EBay and the Management of Possessions as Stock," Journal of Consumer Behaviour, 8, (6), p. 305-315.

Denegri-Knott J., 2011, “'Have It Now!': Ebay and the Acceleration of Consumer Desire”, European Advances in Consumer Reseach, 9, p. 373-379.

Djelassi S., Collin-Lachaud I. et Odou P., 2009, « Crise du pouvoir d'achat. Les distributeurs face au " wising shopping », Décisions marketing, 56, p. 37-46.

Dobscha S., 1998, "The Lived Experience of Consumer Rebellion Against Marketing", Advances in Consumer Research, 25, p. 91-97.

Dolan, P., 2002, "the Sustainability of 'sustainable Consumption'”, Journal of Macromarketing, 22, 2, p. $170-181$.

Durgee J.F. and O'Connor G.C., 1995, “An Exploration into Renting as Consumption Behaviour”, Psychology \& Marketing, 12(2), p. 89-104.

Du Tertre C., 2011, « Modèles économiques d'entreprise, dynamique macroéconomique et développement durable », in Gaglio G et al., L'économie de la fonctionnalité : une voie nouvelle vers un développement durable?, Toulouse, Éditions Octares, p. 21-42.

Etzioni A., 1998, "Voluntary simplicity : Characterization, select psychological implications, and societal consequences", Journal of Economic Psychology, 19, p. 619-643.

European Commission, 2008, Eurobarometer, Attitudes of European citizens towards the environment, Report 295, wage 68.2, March 2008.

Felson M. and Spaeth J. L., 1978, "Community structure and collaborative consumption. A routine activity Approach", The American Behavioral Scientist, 21, 4, p. 614-624.

Fournier S., 1998, "Consumer resistance: societal motivations, consumer manifestations and implications in the marketing domain", Advances in Consumer Research, 25, p. 88-90.

Gaglio G., Lauriol J. et Du Tertre C., 2011, L'économie de la fonctionnalité: une voie nouvelle vers un développement durable? Toulouse, Éditions Octares.

Gansky. L., 2010, The Mesh : Why the future of Business is sharing. New York, Penguin. 
Gleim M.R, Smith J, F, Andrewsb D. and Cronin Jr J.J., 2013, “Against the Green: A Multi-method Examination of the Barriers to Green Consumption", Journal of Retailing, 89 (1), p. 44-61.

Godbout J.T., 1992, L'esprit du don, Éditions La Découverte.

Goedkoop M.J., van Halen C.J.G., te Riele H.R.M. and Rommens P.J.M., 1999, "Product service systems, ecological and economic basis", PricewaterhouseCoopers N.V./Pi !MC, Storrm C.S., PRé Consultants.

Hockerts, K., 1999, “Eco-Efficient Service Innovation: Increasing Business-Ecological Efficiency of Products and Services", in M. Charter (Ed), Greener Marketing : A Global Perspective on Greener Marketing Practice, Sheffield, UK, Greenleaf publishing, p. 95-108

Jackson T., 2005, “Live better by consumer less?" Joumal of Industrial Ecology 19(1/2), p. 19-36.

Jackson, T. and Michaelis, L., 2003, "Policies for Sustainable Consumption”, Sustainable Development Commission, London.

Jackson T., 2010, Prospérité sans croissance, la transition vers une économie durable, Éditions De Boeck.

Kates S.M. and Belk R.W., 2001, “The meanings of Lesbians and Gay Pride day: resistance through consumption and resistance to consumption", Journal of Contemporary Ethnography, 30, 4, p. 392-429.

Katsev R., 2003, “Car Sharing: A New Approach to Urban Transportation Problems," Analyses of Social Issues and Public Policy, 3 (1), p. 65-86.

Kilboume W. E., McDonagh P. B. and Prothero A I., 1997, "Sustainable Consumption and the Quality of Life: A Macromarketing Challenge to the dominant Social Paradigm", Joumal of Macromarketing, 17, 1, p. 4-24.

Kleine S.S., Kleine R.E. and Allen C.T., 1995, "How Is a Possession 'Me' or 'Not Me'? Characterizing Types and an Antecedent of Material Possession Attachment," Journal of Consumer Research, 22, p. 327-343.

Lallement E., 2010, « Ruptures, abandons, déplacements, ethnologie des pratiques alternatives de consommation et d'échange », Sociologies pratiques, 20, p. 23-36.

Lipovetsky G., 2006, Le bonheur paradoxal, Paris, Éditions Gallimard.

Lauriol J., 2008, « Développement durable et économie de la fonctionnalité : une stratégie renouvelée pour de nouveaux enjeux" in L'économie de la fonctionnalité, une voie pour articuler dynamique économique et Développement durable. Enjeux et débats. Publication du Club «Économie de la fonctionnalité et développement durable ", Novembre, p. 11-28.

Lovelock C. and Gummesson E., 2004, "Whither services marketing? In search of a new paradigm and fresh perspectives", Journal of Service Research, 7, 1, p. 20-41.

Luchs M. G., Naylor R. W., Rose R. L., Catlin J. R., Gau R., Kapitan S., et al., 2011, “Toward a sustainable marketplace: Expanding options and benefits for consumers", Journal of Research for Consumers, 19, p. 1-12.

Maffesoli, M., 1988, Le temps des tribus : le déclin de l'individualisme dans les sociétés de masse, Ed Méridiens Klincksieck, Paris.

Markkula A. and Moisander J. K., 2012, "Consumers and discursive confusion over sustainable development", Journal of Consumer Policy, 35, p. 105-125.

Meijkamp R., 1998, “Changing Consumer Behavior Through Eco-efficient Services: An Empirical Study of Car Sharing in The Netherlands", Business Strategy and the Environment, 7, p. 234-244 
Moati P., 2012, La nouvelle révolution commerciale, Paris, Éditions Odile Jacob.

Mont, O., 2004, Product-Service Systems: Panacea or Myth?, Thesis (PhD), The International Institute for Industrial Environmental Economics (IIIEE), Lund University, 259 p.

Mont O., 2002, "Clarifying the concept of product-service system", Journal of Cleaner production, 10, 3, p. 237-245.

Mont O., 2000, Product-Service Systems, Final Report, 288, The International Institute of Industrial Environmental Economics, Lund University, February.

Nelson MR., Rademachcr MA and Paek H-J., 2007, "Downshifting consumer = upshifting citizen? An examination of a local freecycle community", Annals of the American Academy of Political and Social Science 611(1), p. 141-156.

Ostrom. E., 2010, La gouvernance des biens communs : Pour une nouvelle approche des ressources naturelles, Éditions De Boeck.

Ozanne L. and Ballantine P., 2010, "Sharing as a Form of Anti-consumption? An Examination of Toy Library Users", Journal of Consumer Behaviour, 9, (6), p. 485-498.

Peattie K. and Collins A., 2009, "Guest editorial : perspectives on sustainable consumption" International Journal of Consumer Studies, 33, p. 107-112.

Prothero, A. Dobscha, S., Freund, J., Kilbourne, W., Luchs, M. G., Ozanne, L. K. and Thogersen, J., 2011, "Sustainable Consumption: Opportunities for Consumer Research and Public Policy", Journal of Public Policy \& Marketing, 30 (1), p. 31-38.

Reisch, L., 1998, "Sustainable consumption: Three questions about a fuzzy concept" Working paper, Research Group “Consumption Environment and Culture”, Department of Marketing, Copenhagen Business School, Denmark.

Richins M.L., 1994, "Special Possessions and the Expression of Material Values", Journal of Consumer Research, 21, p. 522-533.

Rifkin J., 2012, La troisième révolution industrielle, Paris, Éditions Les liens qui libèrent.

Rifkin J., 2000, L'âge de l'accès, Paris, Ed La Découverte.

Roux, D., 1994, « Les brocantes, réenchantement ou piraterie des systèmes marchands ", Revue française du marketing, 201, 1/5, p. 63-84.

Roy R., 2000, “Sustainable product-service systems”, Futures 32, p. 289-299.

Schneider F., 2009, «Sur l'importance de la décroissance des capacités de production et de consommation dans le Nord Global pour éviter l'Effet Rebond », in Mylondo (Ed), La décroissance économique pour la soutenabilité écologique et l'équité sociale, Collection Ecologica, Éditions du Croquant, p. 197-214.

Schindler R-M., 1989, “The excitement of getting a bargain: some hypotheses concerning the origins and effects of smart-shopper feelings", Advances in consumer research, 16, p. 447-453.

Scholl G., 2006, "Product Service Systems: Taking a Functional and a Symbolic Perspective on Usership" in Maj Munch Andersen and Arnold Tukker (Ed), Perspectives on Radical Changes to Sustainable Consumption and Production (SCP), Workshop of the Sustainable Consumption Research Exchange (SCORE!) Network, 20 and 21 April, Copenhagen, Denmark, p. 25-44.

Schor, J. B., 1998, The Overspent American. Why We Want What We Don't Need, USA, Harper.

Sempels C. et Hoffmann J., 2012, Les business models du futur, Ed Pearson France 
Sheth J. N., Sethia N. K, Srinivas S., 2011, "Mindful consumption : A customer-centric approach to sustainability", Journal of the Academy of Marketing Science, 39, p. 21-39.

Spaargaren, Gert. 2000, "Ecological modernization theory and domestic consumption". Journal of Environmental Policy and Planning, 2, p. 323-335.

Stahel W.R., 1998, "From products to services : selling performance instead of goods", Report vol. 27, Institute for Prospective Technological Studies.

Steininger K., Vogl C. and Zettl R., 1996, “Car-sharing organizations : The size of the market segment and revealed change in mobility behavior", Transport Policy, 3, p. 177-185.

Thøgersen J., 2005, “How May Consumer Policy Empower Consumers for Sustainable Lifestyles ?", Journal of Consumer Policy, 28 (2), p. 143-178.

Vaileanu-Paun I. et Boutillier S., 2012, «Économie de la fonctionnalité, une nouvelle synergie entre le territoire, la firme et le consommateur? ", Innovations, 37, p. 94-125.

Van de Walle I., Hébeln P. et Siounandan N., 2012, « Les secondes vies des objets : les pratiques d'acquisition et de délaissement des produits de consommation ", Cahier de recherche, CREDOC, 290.

Van Niel. J., 2007, L'économie de fonctionnalité: définition et état de l'art, working paper, UTT Université de Lausanne.

Veblen T., 1899, The theory of the leisure class. New York : Modern Library.

Zacklad M. , 2010, « L'économie de la fonctionnalité encastrée dans la socio-économie des transactions coopératives : dynamique servicielle et fidélisation soutenable », in Heurgon E. et Landrieu J. (coord.), L'économie des services pour un développement durable. Nouvelles richesses, nouvelles solidarités, Paris, Éditions L'harmatan, p. 273-288.

\section{NOTES}

1. Expression émanant de Nicolas Le Douarrec, fondateur de CityzenCar, site de location de voitures entre particuliers, Marketing Magazine $\mathrm{N}^{\circ} 153$, novembre 2011, p. 10.

2. Sous le vocable "consommation durable ", nous faisons référence à la terminologie anglosaxonne : "sustainable consumption"

3. http://www.ilokyou.com consulté le 14 octobre 2013.

4. http://www.monjoujou.com, consulté le 25 janvier 2013.

5. http://www.covoiturage.fr/ (site en cours de changement de nom).

6. http://www.covoiturage.fr/ (site en cours de changement de nom).

7. https://www.streetclub.co.uk, consulté le 2 mars 2013.

8. http://www.partagetonfrigo.fr, site consulté le 1er décembre 2013.

9. http://www.covoiturage.fr/ (site en cours de changement de nom).

10. https://www.streetclub.co.uk, consulté le 2 mars 2013.

11. http://www.covoiturage.fr/ (site en cours de changement de nom).

12. ADEME, 2013, Étude «Les Français et les pratiques collaboratives Qui fait quoi ? Et pourquoi ?», 25 avril.

13. Expression de G. Lipovetsky. G. Lipovetsky, 2006, Le bonheur paradoxal, Éditions Gallimard.

14. http://www.lokeo.fr, consulté le 22 avril 2013.

15. http://www.monjoujou.com.

16. https://www.jelouemoncampingcar.com, consulté le 19 décembre 2013.

17. https://www.e-loue.com, consulté le 23 mars 2013. 
18. ADEME, 2013, Étude «Les Français et les pratiques collaboratives Qui fait quoi ? Et pourquoi ?», 25 avril.

19. L'OBSCOLO, 2012, «L'observatoire des consommations émergentes. Evolution des tendances de consommation ", novembre.

20. IFOP pour le compte de CityzenCar, 2011, «Les français prêts à adopter la consommation collaborative! », novembre.

21. Ibid.

22. Exemple des sites tels que E-loue, Lokeo, Mamanloue, Bricolib, Good-look.com, Ma bonne amie, Mon dressing secret, Sac d'un jour...

23. Moati P., 2012, « La consommation collaborative est aussi une manière d'hyperconsommer ", Libération, 10 décembre.

24. http://www.monjoujou.com.

25. http://www.lokeo.fr, consulté le 12 mars 2013.

26. http://www.ecojouet.fr, consulté le 18 mars 2013.

27. https://www.e-loue.com, consulté le 25 avril 2013.

28. http://www.mabonneamie.com, consulté le 12 février 2013.

29. http://www.lafabriquedelunettes.fr, consulté le 2 mars 2013.

\section{RÉSUMÉS}

La consommation collaborative est aujourd'hui portée aux nues dans certains milieux économiques et médiatiques et jugée porteuse de nombreuses vertus tant sur le plan social qu'environnemental. Elle peut être définie comme le versant «consommatoire » de l'économie de la fonctionnalité, son extension naturelle dans le domaine du Business to Consumer (BtoC) ou encore du Consumer to Consumer (CtoC). Cet article explore cette logique de consommation nouvelle et s'interroge sur ses interrelations et sa contribution probable à l'économie de la fonctionnalité dans une logique de durabilité. Il s'appuie sur une analyse de 27 sites internet de consommation collaborative et de blogs consacrés à cette thématique ainsi que sur plusieurs études académiques réalisées sur les services d'usages partagés. Étudiant les ressorts motivationnels identifiés dans la littérature et utilisés pour promouvoir l'usage de ces services collaboratifs auprès des consommateurs, les auteurs clarifient leur contribution et leurs limites en termes de consommation durable.

Collaborative consumption is currently lauded by a number of economists and sections of the media, who extol its many social and environmental merits. It can be defined as the consumerist aspect of the functional economy, its natural extension to the Business-to-Consumer (BtoC) and Consumer-to-Consumer (CtoC) sectors. This article explores this notion of new consumption and examines how it interrelates with and can contribute to the functional economy to develop sustainability. It is based on an analysis of 27 collaborative consumption websites and blogs, together with several academic studies carried out in the field of shared use services. The article studies the reasons and motives that the literature identifies as being used to promote the use of these collaborative services to consumers, and clarifies their contribution and their limits with regard to sustainable consumption. 


\section{INDEX}

Keywords : collaborative consumption, functional economy, sharing, renting, access, nonownership, sustainable consumption

Mots-clés : consommation collaborative, économie de la fonctionnalité, partage, location, accès, non-possession, consommation durable

\section{AUTEURS}

\section{ISABELLE ROBERT}

Isabelle Robert est maître de conférences en sciences de gestion, à l'Institut du Marketing et du Management de la Distribution (IMMD)/Université Lille Nord de France - SKEMA Business School. Ses thématiques de recherche se structurent autour de deux axes : la consommation responsable et la responsabilité sociale de l'entreprise, isabelle.robert-2@univ-lille2.fr

\section{ANNE-SOPHIE BINNINGER}

Docteur en Sciences de Gestion et HDR, Anne-Sophie Binninger est Professeur de Marketing à NEOMA Business School. Elle poursuit ses recherches au sein du groupe de recherche «Marketing, Consommation et Société » et fait partie de l'équipe de recherche transdisciplinaire du LIRSA (CNAM Paris). Ses travaux portent principalement sur les pratiques de consommation durable, anne-sophie.binninger@neoma-bs.fr

\section{NACIMA OURAHMOUNE}

Nacima Ourahmoune est professeur de Marketing à Neoma Business School, où elle co-dirige le centre de recherche « Marketing, Consommation et Société (MCS)». Ses activités de recherche couvrent les thématiques suivantes : communications de marques, consommation et genre, marchés émergents et consommation, approches socioculturelles du marketing et de la consommation, nacima.ourahmoune@neoma-bs.fr 